\title{
QUOTIENTS OF FULLY NONLINEAR CONTROL SYSTEMS*
}

\author{
PAULO TABUADA ${ }^{\dagger}$ AND GEORGE J. PAPPAS ${ }^{\ddagger}$
}

\begin{abstract}
In this paper, we introduce and study quotients of fully nonlinear control systems. Our definition is inspired by categorical definitions of quotients as well as recent work on abstractions of affine control systems. We show that quotients exist under mild regularity assumptions and characterize the structure of the quotient state/input space. This allows one to understand how states and inputs of the quotient system are related to states and inputs of the original system. We also introduce a notion of projectability which turns out to be equivalent to controlled invariance. This allows one to regard previous work on symmetries, partial symmetries, and controlled invariance as leading to special types of quotients. We also show the existence of quotients that are not induced by symmetries or controlled invariance. Such decompositions have a potential use in a theory of hierarchical control based on quotients.
\end{abstract}

Key words. quotient control systems, control systems category, controlled invariance, symmetries

AMS subject classifications. 93A10, 93A30, 93B11, 93C10

DOI. $10.1137 / \mathrm{S} 0363012901399027$

1. Introduction. The analysis and synthesis problems for nonlinear control systems are often very difficult due to the size and complicated nature of the equations describing the processes to be controlled. It is therefore desirable to have a methodology that decomposes control systems into smaller subsystems while preserving the properties relevant for analysis or synthesis. From a theoretical point of view, the problem of decomposing control systems is also extremely interesting since it reveals system structure that must be understood and exploited.

In this paper we will focus on the study of quotient control systems since they can be seen as lower dimensional models that may still carry enough information about the original system. We will build on several accumulated results of different authors that in one way or another have made contributions to this problem. One of the first approaches was given in [17] were the analysis of the Lie algebra of a control system lead to a decomposition into smaller systems. At the same time in [35], quotients of control systems induced by observability equivalence relations where introduced in the more general context of realization theory. In [31], Lie algebraic conditions are formulated for the parallel and cascade decomposition of nonlinear control systems, while the feedback version of the same problem was addressed in [24]. A different approach was based on reduction of mechanical systems by symmetries. In [39], symmetries were introduced for mechanical control systems and further developed in [9] for general control systems. The existence of such symmetries was then used to decompose control systems as the interconnection of lower dimensionality subsystems. The notion of symmetry was further generalized in [26], where it was shown that the existence of symmetries implies that a certain distribution associated with the

${ }^{*}$ Received by the editors December 1, 2001; accepted for publication (in revised form) May 19, 2004; published electronically March 22, 2005. This research was supported by Fundação para a Ciência e Tecnologia under grant PRAXIS XXI/BD/18149/98.

http://www.siam.org/journals/sicon/43-5/39902.html

${ }^{\dagger}$ Department of Electrical Engineering, 268 Fitzpatrick Hall, University of Notre Dame, Notre Dame, IN 46556 (ptabuada@nd.edu).

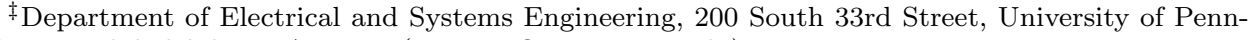
sylvania, Philadelphia, PA 19104 (pappasg@seas.upenn.edu). 
symmetries was controlled invariant. This related the notion of symmetry with the notion of controlled invariance for nonlinear systems. Controlled invariance [23, 12] was also used to decompose systems into smaller components. A different approach was taken in [22] where it was shown how to study controllability of systems evolving on principle fiber bundles through their projection on the base space. More recently, a modular approach to the modeling of mechanical systems has been proposed in [40], by studying how the interconnection of Hamiltonian control systems can still be regarded as a Hamiltonian control system. A different research direction was taken in [29], where instead of using structural properties of control systems, a constructive procedure was proposed to compute smaller control systems called abstractions.

In several of the above approaches, some notion of quotienting is involved. When symmetries exist, one of the blocks of the decompositions introduced in [9] is simply the original control system factored by the action of a Lie group representing the symmetry. If a control system admits a controlled invariant distribution, it is shown in $[23,12]$ that it has a simpler local representation. This simpler representation can be obtained by factoring the original control system by the equivalence relation defined by considering the leaves of the foliation induced by the controlled invariant distribution, equivalence classes. The notion of abstraction introduced in [29] can also be seen as a quotient since the abstraction is a control system on a smaller dimensional state space defined by an equivalence relation on the state space of the original control system. These facts motivate fundamental questions such as existence and characterization of quotient systems. Existence questions have already been addressed in [35] but in a different setting. Only specific equivalence relations were considered (those induced by indistinguishability), and the input space remained unaltered by the factorization process. Furthermore, the quotients discussed in [35] are of a particular nature being characterized by the notion of projectability introduced in section 6 .

A thorough understanding of quotient systems also has important consequences for hierarchical control, since the construction of quotients proposed in [29] implicitly indicates that certain states of the original system may become inputs on the quotient control system. It is perhaps surprising that this methodology interchanges the role of state and input. However, this fact is the crucial factor that allows the development of a hierarchical control theory based on quotients. Since states of the original system may become inputs of the quotient system, a control design performed on a quotient system can serve as a design specification for the original system. We can therefore regard a control design as a specification for the evolution of certain state variables on the more detailed model. A complete and thorough understanding of how the states and inputs propagate from control systems to their quotients will enable such a hierarchical design scheme. Preliminary work exploiting such a hierarchical approach has been reported in [37].

In this paper, we take a new approach to the study of quotients by introducing the category of control systems as the natural setting for such problems in systems theory. The use of category theory for the study of problems in system theory also has a long history which can be traced back to the works of Arbib and Manes (see [2] for an introduction). More recently, several authors have also adopted a categorical approach as in [19], where the category of affine control systems is investigated. We also mention [33], where a categorical approach has been used to provide a general theory of systems.

We define the category of control systems whose objects are fully (nonaffine) nonlinear control systems and morphisms map trajectories between objects. The 
morphisms in this category extend the notion of $\phi$-related systems from [28]. In this categorical setting we formulate the notion of quotient control systems and show, in one of the main results, that

under some regularity (constant rank) assumptions quotient control systems

always exist.

This result implies that, given a nonlinear projection map from the state space to some reduced state space, we can always construct a new control system on the reduced state space with the property that the nonlinear projection map carries trajectories of the original system into trajectories of the reduced system. This should be contrasted with several other approaches which rely on the existence of symmetries or controlled invariance to assert the existence of quotients. We also introduce the notion of projectable control sections, which will be a fundamental ingredient to characterize the structure of quotients. This notion is in fact equivalent to controlled invariance, and this allows one to regard quotients based on symmetries or controlled invariance as a special type of quotients. General quotients, however, are not necessarily induced by symmetries or controlled invariance and have the property that some of their inputs are related to states of the original model. This fact, implicit in [29], is explicitly characterized in this paper by understanding how the state and input space of the quotient is related to the state and input space of the original control system. In particular, this paper's main contribution states that

in the absence of symmetries, states that are factored out in the quotient

construction can be regarded as inputs of the quotient control system.

This result clearly distinguishes general quotients from previously studied quotients based on symmetries or partial symmetries in which inputs of the quotient system are the inputs (or a quotient) of the original system inputs. Since existence of symmetries can be regarded as rare phenomena, ${ }^{1}$ as shown in [32] for single-input systems, construction of quotients enables a widely applicable hierarchical approach to control design based on reconstruction of trajectories for the original system from quotient trajectories [37].

The outline of the paper is as follows. We start by introducing the relevant notions from differential geometry and control theory in section 2 . We then review the notion of $\phi$-related control systems in section 3 which was originally introduced in [28] and will motivate the definition of the category of control systems presented in section 4. In section 5, we introduce the notion of quotient control systems and prove an existence and uniqueness result regarding quotients which roughly asserts that given a regular equivalence relation on the state space of a control system a quotient system exists (under some regularity conditions) and is unique up to isomorphism. The characterization of quotients will be the goal of the remaining sections of the paper. We first introduce the notion of projectable control section in section 6 and prove the main result of the paper characterizing the structure of the quotient state/input space in section 7 . We end with conclusions and some open questions for further research in section 8 .

2. Control systems. In this section we introduce all the relevant notions from differential geometry and control systems necessary for the remainder of the paper. The reader may wish to consult numerous books on these subjects, such as [1] for differential geometry and $[14,27]$ for control theory.

${ }^{1}$ We thank one of the anonymous reviewers for bringing this fact to our attention. 
2.1. Differential geometry. We will consider that all the manifolds will be $C^{\infty}$ and that all the maps will be smooth. Let $M$ be a manifold and $T_{x} M$ its tangent space at $x \in M$. The tangent bundle of $M$ is denoted by $T M=\cup_{x \in M} T_{x} M$, and $\pi_{M}$ is the canonical projection map $\pi_{M}: T M \rightarrow M$ taking a tangent vector $X(x) \in$ $T_{x} M \subset T M$ to the base point $x \in M$. Now, letting $M$ and $N$ be manifolds and $\phi: M$ $\rightarrow N$ a map, we denote by $T_{x} \phi: T_{x} M \rightarrow T_{\phi(x)} N$ the induced tangent map which maps tangent vectors $X$ at $T_{x} M$ to tangent vectors $T_{x} \phi \cdot X$ at $T_{\phi(x)} N$. If $\phi$ is such that $T_{x} \phi$ is surjective at $x \in M$, then we say that $\phi$ is a submersion at $x$. When $\phi$ is a submersion at every $x \in M$ we simply say that it is a submersion. Similarly, we say that $\phi$ has constant rank if the rank of the pointwise linear map $T_{x} \phi$ is constant for every $x \in M$. When $\phi$ has an inverse which is also smooth we call $\phi$ a diffeomorphism. We say that a manifold $M$ is diffeomorphic to a manifold $N$, denoted by $M \cong N$, when there is a diffeomorphism between $M$ and $N$. When this is the case we can use $\phi^{-1}: N \rightarrow M$ to define a vector field on $M$ from a vector field $Y \in T N$, denoted by $\phi^{*} Y=\left(\phi^{-1}\right)_{*} Y$ and defined by $T_{\phi(x)} \phi^{-1} \cdot Y(\phi(x))$.

A fibered manifold is a manifold $B$ equipped with a surjective submersion $\pi_{B}: B$ $\rightarrow M$. Manifolds $B$ and $M$ are called the total space and the base space, respectively. The surjection $\pi_{B}$ defines a submanifold $\pi_{B}^{-1}(x)=\left\{b \in B: \pi_{B}(b)=x\right\} \subseteq B$ for every $x \in M$ called the fiber at $x \in M$. We will usually denote a fibered manifold simply by $\pi_{B}: B \rightarrow M$. Since a surjective submersion is locally the canonical projection from $\mathbb{R}^{i}$ to $\mathbb{R}^{j}, i=\operatorname{dim}(B)$ and $j=\operatorname{dim}(M)$, we can always find local coordinates $(x, y)$, where $x$ are coordinates for the base space and $y$ are coordinates for the fibers over the base space. We shall call these coordinates adapted coordinates.

A map $\varphi: B_{1} \longrightarrow B_{2}$ between two fibered manifolds is fiber preserving iff there exists a map $\phi: M_{1} \rightarrow M_{2}$ between the base spaces such that the following diagram commutes:

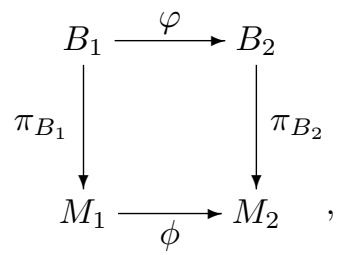

that is to say, iff $\pi_{B_{2}} \circ \varphi=\phi \circ \pi_{B_{1}}$. In such a case we also refer to $\varphi$ as a fiber preserving lift of $\phi$. Given fibered manifolds $B_{1}$ and $B_{2}$, we will say that $B_{1}$ is a fibered submanifold of $B_{2}$ if the inclusion map $i: B_{1} \hookrightarrow B_{2}$ is fiber preserving.

Given a map $h: M \rightarrow N$ defined on the base space of a fibered manifold, its extension to the total space $B$ is given by $\pi_{B}^{*} h=h \circ \pi_{B}$. We now consider the extension of a map $H: B \rightarrow T M$ to a vector field in $B$. We will define local and global extensions of $H$. Globally, we define $H^{e}$ as the set of all vector fields ${ }^{2} X: B$

\footnotetext{
${ }^{2}$ Global existence of such vector fields $X$ follows from the existence of a horizontal space $\mathcal{H} \subseteq T B$, $\mathcal{H} \cong T M$ that allows the decomposition of $T B$ as $T B=\mathcal{H} \oplus \operatorname{ker}\left(T \pi_{B}\right)$. A global extension of a map $H: B \rightarrow T M$ to a vector field $X: B \rightarrow T B$ is now uniquely defined as the vector field $X=H: B \rightarrow T M \cong \mathcal{H} \subseteq T B$. Such horizontal space can be obtained, for example, as the orthogonal complement to $\operatorname{ker}\left(T \pi_{B}\right)$ given by a Riemannian metric on $B$.
} 
$\rightarrow T B$ such that the following diagram commutes:

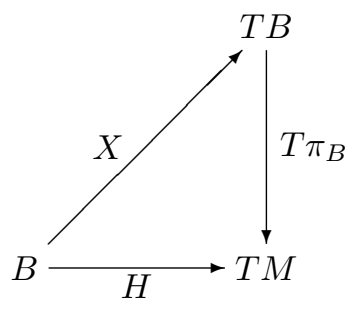

that is, $T \pi_{B}(X)=H$. When working locally, one can be more specific and select a distinguished element of $H^{e}$, denoted by $H^{l}$, which satisfies in adapted local coordinates $(x, y), H^{l}=H \frac{\partial}{\partial x}+0 \frac{\partial}{\partial y}$. A vector field $Y: M \longrightarrow T M$ on the base space $M$ of a fibered manifold can also be extended to a vector field on the total space. It suffices to compose $Y$ with the projection $\pi_{B}: B \rightarrow M$ and recover the previous situation since $Y \circ \pi_{B}$ is a map from $B$ to $T M$.

2.2. Control systems. Since the early days of control theory it was clear that in order to give a global definition of control systems the notion of input could not be decoupled from the notion of state $[4,41]$. Although the coupling between states and inputs is usually modeled through the use of fiber bundles, we shall consider more general spaces.

In any case a control system can be globally defined as follows.

Definition 2.1 (control system). A control system $\Sigma_{M}=\left(U_{M}, F_{M}\right)$ consists of a fibered manifold $\pi_{U_{M}}: U_{M} \longrightarrow M$ called the control bundle and a map $F_{M}: U_{M}$ $\longrightarrow T M$ making the following diagram commutative:

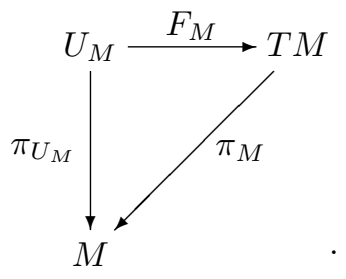

That is, $\pi_{M} \circ F_{M}=\pi_{U_{M}}$, where $\pi_{M}: T M \rightarrow M$ is the tangent bundle projection.

The input space $U_{M}$ is modeled as a fibered manifold since in general the available control inputs may depend on the current state of the system. In adapted coordinates $(x, v)$, Definition 2.1 reduces to the familiar expression $\dot{x}=f(x, v)$ with $v \in \pi_{U_{M}}^{-1}(x)$. The lack of local triviality assumptions on $\pi_{U_{M}}$ is motivated by the need to model the construction of abstractions of control affine systems, as described in [29], in a fully nonlinear context. As the following example illustrates, even in simple situations the inputs of a control system resulting from an abstraction or quotient process can depend on the states in a way that cannot be modeled by a fiber bundle.

Consider control system $F_{M}: U_{M} \rightarrow T M$ with $\left.U_{M}=M \times U, M=\mathbb{R}^{3}, U=\right] 0,1[$ defined by:

$$
F_{M}(x, y, z, u)=\left(x \frac{\partial}{\partial x}+y \frac{\partial}{\partial y}+z \frac{\partial}{\partial z}\right) u .
$$

On the state space we define the following map $\phi: \mathbb{R}^{3} \rightarrow \mathbb{R}$ based on Reeb's foliation:

$$
\phi(x, y, z)=\left(1-r^{2}\right) e^{z}, \quad r=x^{2}+y^{2} .
$$


Computing the derivative of $\phi$,

$$
\mathrm{d} \phi=e^{z}\left(-4 r x \mathrm{~d} x-4 r y \mathrm{~d} y+\left(1-r^{2}\right) \mathrm{d} z\right)
$$

we see that $\phi$ is a submersion since $1-r^{2}=0$ for $r^{2}=1$, which implies that $x \neq 0$ or $y \neq 0$, and this in turn implies that $\mathrm{d} \phi \neq 0$. This shows that we can see $\phi: \mathbb{R}^{3} \rightarrow \mathbb{R}$ as a fibered manifold. If we now compute the projection of $F_{M}$ on $\mathbb{R}$ by $\phi$, we obtain

$$
\mathrm{d} \phi \cdot F_{M}=e^{z}\left(-4 r x^{2}-4 r y^{2}+\left(1-r^{2}\right) z\right) u .
$$

The set of vectors defined by the previous expression can be seen as a control system on $\mathbb{R}$ up to control parameterization, as it defines the possible directions of motion achievable by control. This is the principle underlying the notion of abstraction described in [29]. Such a collection of vector fields admits the natural parameterization $\pi_{U_{M}}^{-1}\left(\phi^{-1}(w)\right)$ for every $w \in \mathbb{R}$. However, such a set of inputs cannot be given the structure of a fiber bundle. To see this, it suffices to note that the fibers $\phi^{-1}(w)$ are not homeomorphic for $w>0$ and $w=0$. For $w>0$ we can solve $\phi(x, y, z)=w$ to obtain $z=\log \frac{w}{1-r^{2}}$ which defines $\phi^{-1}(w)$ as

$$
\left\{(x, y, z) \in \mathbb{R}^{3}: z=\log \frac{w}{1-r^{2}} \wedge 0 \leq r^{2}<1\right\}
$$

and which is homeomorphic to the open unit disk in $\mathbb{R}^{2}$. If $w=0$, solving $\phi(x, y, z)=$ 0 , we obtain $r=1$ which is diffeomorphic to a cylinder. We thus see that for any open set $O$ in $\mathbb{R}$ containing $0, \pi_{U_{M}}\left(\phi^{-1}(0)\right)$ cannot be diffeomorphic to $O \times L$ for some manifold $L$ describing the typical fibers of $\phi \circ \pi_{U_{M}}$ as they are not diffeomorphic for different points in $O$. It is precisely the need to capture and analyze situations like this that forces one to consider models for the state/input space other than fiber bundles. The need to model these and other couplings between states and inputs has led to alternative approaches where the notion of control system and its properties are defined independently of states and inputs as in Willem's behavioral theory [30] and Fliess' differential algebraic approach [7].

We now return to our discussion of control systems by introducing the notion of control section ${ }^{3}$ that is closely related to control systems and which will be fundamental in our study of quotients.

Definition 2.2 (control section). Given a manifold $M$, a control section on $M$ is a fibered submanifold $\pi_{\mathcal{S}_{M}}: \mathcal{S}_{M} \longrightarrow M$ of $T M$.

We denote by $\mathcal{S}_{M}(x)$ the set of vectors $X \in T_{x} M$ such that $X \in \pi_{\mathcal{S}_{M}}^{-1}(x)$. We now see that under certain regularity assumptions, a control system $\left(U_{M}, F_{M}\right)$ defines a control section by the pointwise assignment $\mathcal{S}_{M}(x)=F_{M}\left(\pi_{U_{M}}^{-1}(x)\right)$. Conversely, a control section also defines a control system as we shall see in detail in section 4 . The notion of a control section allows one to refer in a concise way to the set of all tangent vectors that belong to the image of $F_{M}$ by saying that $X \in T_{x} M$ belongs to $\mathcal{S}_{M}(x)$ iff there exists a $u \in U_{M}$ such that $\pi_{M}(u)=x$ and $F_{M}(u)=X$. When $\mathcal{S}_{M}(x)$ defines an affine distribution on $T M$, we call control system $F_{M}$ control affine and fully nonlinear otherwise.

Having defined control systems the concept of trajectories or solutions of a control system is naturally expressed by the following definition.

\footnotetext{
${ }^{3}$ In some literature this notion is also known as the field of admissible velocities.
} 
Definition 2.3 (trajectories of control systems). A smooth curve $c: I \rightarrow M$, $I \subseteq \mathbb{R}_{0}^{+}$, is called a trajectory of control system $\Sigma_{M}=\left(U_{M}, F_{M}\right)$ if there exists a (not necessarily smooth) curve $c^{U}: I \longrightarrow U_{M}$ making the following diagrams commutative:
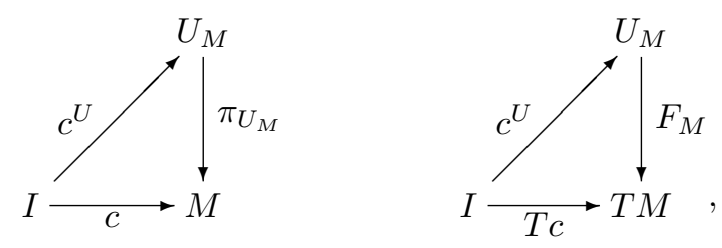

where we have identified I with TI.

The above commutative diagrams are equivalent to the following equalities:

$$
\begin{aligned}
\pi_{U_{M}} \circ c^{U} & =c, \\
T c & =F_{M}\left(c^{U}\right),
\end{aligned}
$$

which mean in adapted coordinates that $x(t)$ is a trajectory of a control system if there exists an input $v(t)$ such that $x(t)$ satisfies $\dot{x}(t)=f(x(t), v(t))$ and $v(t) \in \pi_{U_{M}}^{-1}(x(t))$ for all $t \in I$.

3. $\phi$-related control systems. We start by reviewing the notion of $\phi$-related control systems originally introduced in [28] and which motivates the construction of the category of control systems to be later presented.

DeFinition 3.1 ( $\phi$-related control systems). Let $\Sigma_{M}$ and $\Sigma_{N}$ be two control systems defined on manifolds $M$ and $N$, respectively. Given a map $\phi: M \rightarrow N$ we say that $\Sigma_{N}$ is $\phi$-related to $\Sigma_{M}$ iff for every $x \in M$,

$$
T_{x} \phi\left(\mathcal{S}_{M}(x)\right) \subseteq \mathcal{S}_{N} \circ \phi(x) .
$$

In [28] it is shown that this notion is equivalent to a more intuitive relation between $\Sigma_{M}$ and $\Sigma_{N}$.

Proposition 3.2 (see [28]). Let $\Sigma_{M}$ and $\Sigma_{N}$ be two control systems defined on manifolds $M$ and $N$, respectively, and let $\phi: M \longrightarrow N$ be a map. Control system $\Sigma_{N}$ is $\phi$-related to $\Sigma_{M}$ iff for every trajectory $c(t)$ of $\Sigma_{M}, \phi(c(t))$ is a trajectory of $\Sigma_{N}$.

Propagating trajectories from one system to another is clearly desirable. Since most control system properties are properties of its trajectories, relating trajectories of different control systems also allows one to relate the corresponding properties. If, in fact, system $\Sigma_{N}$ is lower dimensional than system $\Sigma_{M}$, then we are clearly reducing the complexity of $\Sigma_{M}$. We can therefore regard $\Sigma_{N}$ as an abstraction of $\Sigma_{M}$ in the sense that some aspects of $\Sigma_{M}$ have been collapsed or abstracted away, while others remain in $\Sigma_{N}$. This motivated the notion of abstraction based on trajectory propagation in [28], which defined an abstraction of a control system $\Sigma_{M}$ as a $\phi$-related control system $\Sigma_{N}$ by a surjective submersion $\phi$.

The idea of sending trajectories from one system to trajectories of another system has been used many times in control theory to study equivalence of control systems. We mention, for example, linearization by diffeomorphism [16] or feedback linearization $[5,10,13]$. In these examples the maps $\phi$ relating the control systems were in fact diffeomorphisms so that no aggregation or abstraction was involved. Related to the feedback linearization problem is the partial feedback linearization problem where only partial linearization is thought of. Such a problem can be reduced to the feedback linearization problem by considering feedback linearization of a subsystem of 
the original control system [20]. The notion of a subsystem can also be described by defining how subsystem trajectories relate to the original system trajectories. In this case, we require the existence of a map (satisfying certain injectivity assumptions) transforming subsystem trajectories into trajectories of the original system. The use of trajectory propagating maps can already be traced back to the works of Arbib and Manesl (see [2] for an introduction), where by the use of category theoretic ideas it is shown that (discrete time) control systems and finite state automata are just different manifestations of the same phenomena.

4. The category of control systems. Informally speaking, a category is a collection of objects and morphisms between the objects that relate to the structure of the objects. If one is interested in understanding vector spaces, it is natural to consider vector spaces as objects and linear maps as morphisms, since they preserve the vector space structure. This choice for objects and morphisms defines Vect, the category of vector spaces. Choosing manifolds for objects leads to the natural choice of smooth maps for morphisms and defines Man, the category of smooth manifolds. In this section we introduce the category of control systems which we regard as the natural framework to study quotients of control systems. Besides providing an elegant language to describe the constructions to be presented, category theory also offers a conceptual methodology for the study of objects, control systems in this case. We refer the reader to [18] and [3] for further details on the elementary notions of category theory used throughout the paper.

The category of control systems, denoted by Con, has as objects control systems as described in Definition 2.1. The morphisms in this category extend the concept of $\phi$-related control systems described by Definition 3.1. Since the notion of $\phi$-related control systems relates control sections and these can be parameterized by controls, the lifted notion should relate control sections as well as its parameterizations by inputs.

Definition 4.1 (morphisms of control systems). Let $\Sigma_{M}$ and $\Sigma_{N}$ be two control systems defined on manifolds $M$ and $N$, respectively. A morphism $f$ from $\Sigma_{M}$ to $\Sigma_{N}$ is a pair of maps $f=(\phi, \varphi), \phi: M \longrightarrow N$ and $\varphi: U_{M} \longrightarrow U_{N}$ making the following diagrams commutative:
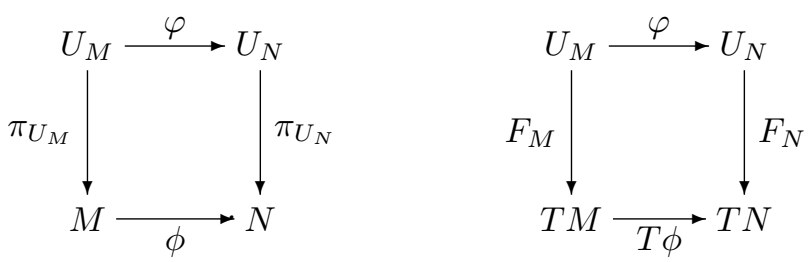

It will be important for later use to also define isomorphisms.

DeFinition 4.2 (isomorphisms of control systems). Let $\Sigma_{M}$ and $\Sigma_{N}$ be two control systems defined on manifolds $M$ and $N$, respectively. System $\Sigma_{M}$ is isomorphic to system $\Sigma_{N}$ iff there exist morphisms $f_{1}$ from $\Sigma_{M}$ to $\Sigma_{N}$ and $f_{2}$ from $\Sigma_{N}$ to $\Sigma_{M}$ such that $f_{1} \circ f_{2}=\left(i d_{N}, i d_{U_{N}}\right)$ and $f_{2} \circ f_{1}=\left(i d_{M}, i d_{U_{M}}\right)$.

In this setting, feedback transformations ${ }^{4}$ can be seen as special isomorphisms. Consider an isomorphism $(\phi, \varphi)$ with $\varphi: U_{M} \rightarrow U_{M}$ such that $\phi=i d_{M}$. In adapted

\footnotetext{
${ }^{4}$ Some authors use the expression feedback transformation to denote any isomorphism in Con. We consider the more restrictive use where changes of coordinates in the state space are disallowed as they cannot be realized by feedback.
} 
coordinates $(x, v)$, where $x$ represents the base coordinates (the state) and $v$ the coordinates on the fibers (the inputs), the isomorphism has a coordinate expression for $\varphi$ of the form $\varphi=(x, \beta(x, v))$. The fiber term $\beta(x, v)$ representing the new control inputs is interpreted as a feedback transformation since it depends on the state at the current location as well as the former inputs $v$. We shall therefore refer to feedback transformations as isomorphisms over the identity since we have $\phi=i d_{M}$. The control theoretic notion of feedback equivalence is captured in this framework by noting that two control systems are feedback equivalent iff there exists an isomorphism (although not necessarily a feedback transformation) between the two systems. A related notion is that of system immersion. Although we cannot capture such a notion in our framework, as we have not equipped control systems with observation maps, a restricted version of system immersion can still be defined within our framework. Recall that, according to [6], system $\Sigma_{M}$ is said to be immersed in system $\Sigma_{N}$ if there exists an injective map $\phi: M \rightarrow N$ such that the input-output behavior of $\Sigma_{M}$, when initialized at $x$, equals the input-output behavior of $\Sigma_{N}$, when initialized at $\phi(x)$. If we assume that $U_{M}=U \times M$ and $U_{N}=U \times N$ for some common input manifold $U$, that $M$ is a submanifold of $N$, and that $i$ is the canonical injection of $M$ into $N$, then $\Sigma_{M}$ is immersed into $\Sigma_{N}$, when $\left(i d_{U}, i\right)$ is a morphism from $\Sigma_{M}$ to $\Sigma_{N}$. Note that the existence of morphism $\left(i d_{U}, i\right)$ implies that $F_{M}(x, u)=T i \cdot F_{M}(x, u)=$ $F_{N}\left(i(x), i d_{U}(u)\right)=F_{N}(x, u)$ for local coordinates $(x, u) \in U \times M \subseteq U \times N$, and this implies that $\Sigma_{M}$ and $\Sigma_{N}$ have the same input-output behavior when initialized at $x$ and $i(x)$, respectively.

A control system can alternatively be defined by a control section $\mathcal{S}_{M}$ on $M$ in the sense that at each point $x \in M, \mathcal{S}_{M}(x)$ defines all the possible directions along which we can flow or steer our system. However, there can be several control parameterizations for $\mathcal{S}_{M}$ and it is important to understand in what sense all those parameterizations represent the same control system. In order to obtain such equivalence we make the following assumptions about control systems that will be explicitly mentioned when needed:

AI: The fibers $\pi_{U_{M}}^{-1}(x)$ are connected for every $x \in M$.

AII: The map $F_{M}: U_{M} \longrightarrow T_{M}$ is an embedding.

Control systems satisfying assumption AII enjoy the following property.

Proposition 4.3. Let $\left(U_{M}, F_{M}\right)$ be a control system on manifold $M$ satisfying AII and let $\left(U_{M}^{\prime}, F_{M}^{\prime}\right)$ be any control system on manifold $M$ such that $\mathcal{S}_{M}^{\prime}(x) \subseteq \mathcal{S}_{M}(x)$ for every $x \in M$. Then, there exists a unique fiber preserving map $\overline{F_{M}}$ making the following diagram commutative:

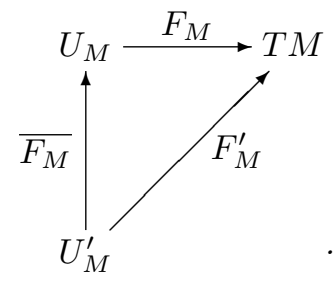

The previous result is an immediate consequence of the fact that $F_{M}\left(U_{M}\right)$ is an embedded submanifold of $T M$. This is sufficient for the previous result to hold but not necessary. In fact, the existence of a unique map $\overline{F_{M}}$ is the property of interest and could be used as a definition. However, it would be difficult to check in concrete examples if a given control system would satisfy such a property. A different approach 
would relax the requirement that $F_{M}\left(U_{M}\right)$ is an embedded submanifold by the weaker assumption of initial submanifold (see [15] for the definition of initial submanifolds and its properties).

Since assumption AII implies the universal property [18] stated in Proposition 4.3, any two control systems satisfying AII and defining the same control section are isomorphic. It is in this sense that we do not need to distinguish between different parameterizations of the same control section. They are the same control system, up to a change of control coordinates, that is, up to an isomorphism over the identity. This will be important when considering the effect of feedback since, as we have already seen, this change of control coordinates can be regarded as a feedback transformation.

The relation between the notions of $\phi$-related control systems (3.1) and Con morphisms (4.1) is stated in the next proposition.

Proposition 4.4. Let $\Sigma_{M}$ and $\Sigma_{N}$ be two control systems defined on $M$ and $N$, respectively. If $f=(\phi, \varphi)$ is a Con morphism from $\Sigma_{M}$ to $\Sigma_{N}$, then $\Sigma_{N}$ is $\phi$-related to $\Sigma_{M}$. Conversely, if $\Sigma_{N}$ satisfies AII and $\Sigma_{N}$ is $\phi$-related to $\Sigma_{M}$ by a smooth map $\phi: M \rightarrow N$, then there exists a unique fiber preserving lift $\varphi$ of $\phi$ such that $f=(\phi, \varphi)$ is a Con morphism from $\Sigma_{M}$ to $\Sigma_{N}$.

Proof. Definition 4.1 trivially implies Definition 3.1, so let us prove that Definition 3.1 implies Definition 4.1. If $\Sigma_{N}$ is $\phi$-related to $\Sigma_{M}$, then by Definition 3.1, $T_{x} \phi\left(\mathcal{S}_{M}(x)\right) \subseteq \mathcal{S}_{N} \circ \phi(x)$. But $\mathcal{S}_{M}$ is parameterized by $U_{M}$, so that the map $T \phi \circ F_{M}: U_{M} \longrightarrow T N$ (see the diagram below) satisfies $T \phi \circ F_{M}\left(U_{M}\right) \subseteq \mathcal{S}_{N}$. Therefore, by Proposition 4.3, there is a unique fiber preserving map $\overline{F_{N}}$ such that

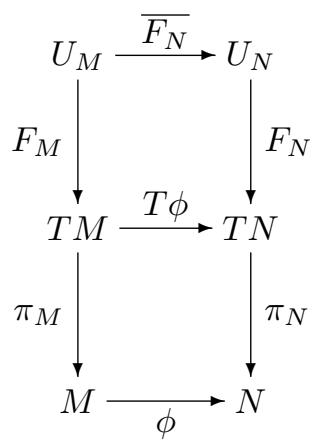

commutes. By taking $\varphi=\overline{F_{N}}, \pi_{U_{M}}=\pi_{M} \circ F_{M}$, and $\pi_{U_{N}}=\pi_{N} \circ F_{N}$ one recovers Definition 4.1 and the equivalence is proved.

The previous result shows that there is an equivalence between smooth maps $\phi$ relating control systems and Con morphisms provided that we work on a suitable subcategory (where assumption AII holds). This means that many properties of nonlinear control systems can be characterized by working with $\mathcal{S}_{M}$ instead of $F_{M}$. We also see that if there is a morphism $f$ from $\Sigma_{M}$ to $\Sigma_{N}$, then this morphism carries trajectories of $\Sigma_{M}$ to trajectories of $\Sigma_{N}$ in virtue of Proposition 3.2.

5. Quotients of control systems. Given a control system $\Sigma_{M}$ and an equivalence relation on the manifold $M$, we can regard the quotient control system as an abstraction since some modeling details propagate from $\Sigma_{M}$ to the quotient while other modeling details disappear in the factorization process. This fact motivates the study of quotient control systems as they represent lower complexity (dimension) objects that can be used to verify properties of the original control system. Quotients are also important from a design perspective since a control law for the quotient object can be regarded as a specification for the desired behavior of the original control 
system. In this spirit we will address the following questions.

1. Existence. Given a control system $\Sigma_{M}$ defined on a manifold $M$ and an equivalence relation $\sim_{M}$ on $M$, when does there exist a control system on $M / \sim_{M}$, the quotient manifold, and a fiber preserving lift $p_{U}$ of the projection $p_{M}: M \rightarrow M / \sim_{M}$ such that $\left(p_{M}, p_{U}\right)$ is a Con morphism?

2. Uniqueness. Is the lift $p_{U}$ of $p_{M}$, when it exists, unique?

3. Structure of the quotient state/input space. What is the structure of the fibers (input space) of the quotient control system?

To clarify our discussion we formalize the notion of quotient control systems.

DeFinition 5.1 (quotient control system). Let $\Sigma_{L}, \Sigma_{M}, \Sigma_{N}$ be control systems defined on manifolds $L, M$, and $N$, respectively, and $g$, $h$ two morphisms from $\Sigma_{L}$ to $\Sigma_{M}$. The pair $\left(f, \Sigma_{N}\right)$, where $f$ is a morphism and $\Sigma_{N}$ a control system, is a quotient control system of $\Sigma_{M}$ if $f \circ g=f \circ h$ and for any other pair $\left(f^{\prime}, \Sigma_{N}^{\prime}\right)$ such that $f^{\prime} \circ g=f^{\prime} \circ h$ there exists one and only one morphism $\bar{f}$ from $\Sigma_{N}$ to $\Sigma_{N}^{\prime}$ such that the following diagram commutes:

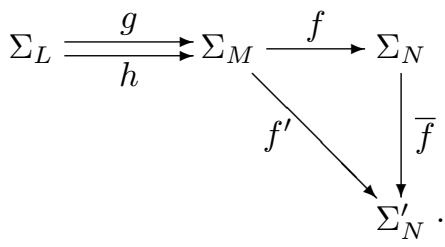

That is, $f^{\prime}=\bar{f} \circ f$.

Intuitively, we can read diagram (5.1) as follows. Assume that the set $\sim=$ $\left\{(u, v) \in U_{M} \times U_{M} \quad: \quad(u, v)=(g(l), h(l))\right.$ for some $\left.l \in U_{L}\right\}$ is a regular equivalence relation [1]. Then, the condition $f \circ g=f \circ h$ simply means that $f$ respects the equivalence relation, that is, $u \sim v \Rightarrow f(u)=f(v)$. Furthermore, it asks that for any other map $f^{\prime}$ respecting relation $\sim$, there exists a unique map $\bar{f}$ such that $f^{\prime}=\bar{f} \circ f$. This is a usual characterization of quotient manifolds [1] that we use here as a definition. The same chain of reasoning shows that if we replace control systems by the corresponding state space and the morphisms by the maps between the state spaces, then diagram (5.1) asks for $N$ to also be quotient manifold obtained by factoring $M$ by a regular equivalence relation $\sim_{M}$ on $M$ defined by $g$ and $h$. The same idea must therefore hold for control systems. This means that control system $\Sigma_{N}$ must also satisfy a unique factorization property in order to be a quotient control system.

From the above discussion it is clear that a necessary condition for the existence of the quotient control system is the existence of the quotient manifold $M / \sim_{M}$. When $\sim_{M}$ is a regular equivalence relation the quotient space $M / \sim_{M}$ will be a manifold [1] and the equivalence relation can be equivalently described by a surjective submersion. We will therefore assume that the regular equivalence relation $\sim_{M}$ is given by a surjective submersion $\phi: M \rightarrow N$. Similarly, the fiber preserving lift $\varphi$ of $\phi$ will also have to be a surjective submersion. We now consider the following assumption which will be explicitly stated when required.

AIII: The map $T \phi \circ F_{M}: U_{M} \longrightarrow T N$ has constant rank and connected fibers.

The first two questions of the previous list are answered in the next theorem which asserts that quotients exist under moderate conditions.

THEOREM 5.2. Let $\Sigma_{M}$ be a control system on a manifold $M$ and $\phi: M \rightarrow N$ a surjective submersion, and assume that AIII holds. Then there exist,

1. a control system $\Sigma_{N}$ on $N$, 
2. a unique fiber preserving lift $\varphi: U_{M} \longrightarrow U_{N}$ of $\phi$ such that the pair $\left((\phi, \varphi), \Sigma_{N}\right)$ is a quotient control system of $\Sigma_{M}$.

Proof. By assumption AIII, the map $T \phi \circ F_{M}$ has constant rank and we can define a regular and involutive distribution $\mathcal{D}$ on $T U_{M}$ by $\mathcal{D}=\operatorname{ker}\left(T T \phi \circ T F_{M}\right)$. Furthermore, as $T \phi \circ F_{M}$ has connected fibers, also by assumption AIII, these are described by the integral manifolds of $\mathcal{D}$. We thus have a regular equivalence relation $\sim \subseteq U_{M} \times U_{M}$ obtained by declaring two points equivalent if they lie on the same integral manifold of $\mathcal{D}$. We now consider the manifold $U_{M} / \sim$ obtained as the quotient of $U_{M}$ by $\sim$ and denote by $\pi: U_{M} \rightarrow U_{M} / \sim$ the canonical projection. Since $T \phi \circ F_{M}(u)=T \phi \circ F_{M}\left(u^{\prime}\right)$ iff $\pi(u)=\pi\left(u^{\prime}\right)$, it follows from the properties of quotient manifolds [1] that there exists a unique map $\alpha: U_{M} / \sim T N$ such that $\alpha \circ \pi=$ $T \phi \circ F_{M}$. We now define $U_{N}$ as $U_{M} / \sim, \pi_{U_{N}}$ as $\pi_{N} \circ \alpha, F_{N}$ as $\alpha$, and $\varphi$ as $\pi$. We note that $\varphi$ is unique and claim that $\left((\phi, \varphi), U_{M} / \sim\right)$ is a quotient of $\Sigma_{M}$. The pair of maps $(\phi, \varphi)$ is a morphism from $\Sigma_{M}$ to $\Sigma_{N}$ since $T \phi \circ F_{M}=F_{N} \circ \varphi$ as required by the second diagram in (4.1), and composing $T \phi \circ F_{M}=F_{N} \circ \varphi$ with $\pi_{N}$, we obtain,

$$
\begin{array}{rlr}
\pi_{N} \circ T \phi \circ F_{M} & =\pi_{N} \circ F_{N} \circ \varphi & \\
\Leftrightarrow \phi \circ \pi_{M} \circ F_{M}=\pi_{N} \circ F_{N} \circ \varphi & \text { since } \pi_{N} \circ T \phi=\phi \circ \pi_{M} \\
\Leftrightarrow \phi \circ \pi_{U_{M}}=\pi_{N} \circ F_{N} \circ \varphi & \text { by commutativity of diagram (2.3) } \\
\Leftrightarrow \phi \circ \pi_{U_{M}}=\pi_{U_{N}} \circ \varphi & \text { by definition of } \pi_{U_{N}},
\end{array}
$$

which shows that the first diagram in (4.1) also commutes.

It remains to show that any other morphism $f^{\prime}=\left(\phi^{\prime}, \varphi^{\prime}\right)$ such that $\phi^{\prime}$ is compatible with the equivalence relation defined by $\phi$ factors uniquely through $f$. Since the equivalence relation defined by $\phi$ on $M$ induces the equivalence relation $\sim$ on $U_{M}$, we see that $\varphi(u)=\varphi\left(u^{\prime}\right)$ implies $\varphi^{\prime}(u)=\varphi^{\prime}\left(u^{\prime}\right)$. It then follows from the universality of $\varphi$ that $\varphi^{\prime}$ factors uniquely through $\varphi$; that is, there exists a unique map $\bar{\varphi}$ such that $\varphi^{\prime}=\bar{\varphi} \circ \varphi$. Similarly, $\phi^{\prime}$ factors uniquely trough $\phi$ via $\bar{\phi}$. It then remains to show that $(\bar{\phi}, \bar{\varphi})$ is a morphism from $\Sigma_{N}$ to $\Sigma_{N}^{\prime}$.

We first show that diagram (4.1) commutes. Let $u_{n} \in U_{N}$, as $\varphi$ is a surjective map; there is a $u_{m} \in U_{M}$ such that $\varphi\left(u_{m}\right)=u_{n}$. We now have, by diagram chasing,

$$
\begin{aligned}
F_{N}^{\prime} \circ \bar{\varphi} \circ \varphi\left(u_{m}\right)=F_{N}^{\prime} \circ \varphi^{\prime}\left(u_{m}\right) & \text { since } \varphi^{\prime} \text { factors on } \varphi \\
=T \phi^{\prime} \circ F_{M}\left(u_{m}\right) & \text { by commutativity of the 2nd diagram in (4.1) } \\
=T \bar{\phi} \circ T \phi \circ F_{M}\left(u_{m}\right) & \text { since } \phi^{\prime} \text { factors on } \phi \\
=T \bar{\phi} \circ F_{N} \circ \varphi\left(u_{m}\right) & \text { by commutativity of the 2nd diagram in (4.1), }
\end{aligned}
$$

and replacing $\varphi\left(u_{m}\right)$ by $u_{n}$ we see that $\bar{f}$ satisfies the second diagram in (4.1). Commutativity of the first diagram in (4.1) can be obtained similarly by diagram chasing.

This result provides the first characterization of quotient objects in Con. It shows that given a regular equivalence relation on the base (state) space of a control system and a mild regularity condition, there exists a quotient control system on the quotient manifold. Furthermore, it also shows that the regular equivalence relation on $M$ or the map $\phi$ uniquely determines a fiber preserving lift $\varphi$ which describes how the state/input pairs of the control system on $M$ relate to the state/input pairs of the quotient control system. Furthermore, we also see that the map $F_{N}$ is an injective immersion, a fact we will use several times in the remainder of this paper.

Existence of quotients under such weak conditions is perhaps surprising given the fact that in other contexts, quotients exist only in very specific situations. A quotient 
group can only be obtained by factoring a group by a normal subgroup and not by a general equivalence relation, a quotient linear space can only be obtained by factoring a linear space by a linear subsubspace and not by a general equivalence relation, etc. This fact highlights the relevance of Theorem 5.2 at the theoretical level but also at the practical level since quotients can be constructively used to hierarchically design trajectories [37].

Having answered the first two questions from the previous list (existence and uniqueness), we concentrate on the characterization of the quotient control system input space. This problem requires a deeper understanding of how $\phi$ determines $\varphi$ and will be the goal of the remainder of this paper. Since Con was defined over Man, that is, morphisms in Con are smooth maps and control systems are defined on manifolds, the characterization of $\varphi$ will require an interplay of tools from differential geometry and category theory.

6. Projectable control sections. We now extend the notion of projectable vector fields from [21] and of projectable families of vector fields from [22] to control sections. The notion of projectable control sections is weaker than projectable vector field or families of vector fields but nonetheless stronger than Con morphisms. The motivation for introducing this notion comes from the fact that projectability of control sections will be a fundamental ingredient in characterizing the structure of the quotient system input space . Furthermore, we will also see that projectability, as defined in this categorical setting, will correspond to the well-known notion of controlled invariance.

Given a vector field $X$ on $M$ and a surjective submersion $\phi: M \rightarrow N$ we say that $X$ is projectable with respect to $\phi$ when $Y=T \phi \cdot X$, the projection of $X$, is a well-defined vector field on $N$ that satisfies $T \phi \cdot X=Y \circ \phi$ [21]. The vector field $Y$ is also called $\phi$-related to $X[1]$. This notion was extended to families of vector fields in [22] by requiring that the projection of each vector field in the family is a well-defined vector field on $N$. However, when working with control sections, which can be regarded as sets of vectors at each base point $x \in M$, one should require only that the projection of these sets of vectors is the same set when the base points on $M$ project on the same base point on $N$. Intuitively, we are asking for control sections that behave well under the projection defined by $\phi$. This is formalized as follows.

DeFINITION 6.1. Let $M$ be a manifold, $\mathcal{S}_{M}$ a control section on $M$, and $\phi: M$ $\rightarrow N$ a surjective submersion. We say that $\mathcal{S}_{M}$ is projectable with respect to $\phi$ iff $\mathcal{S}_{M}$ induces a control section $\mathcal{S}_{N}$ on $N$ such that the following diagram commutes:

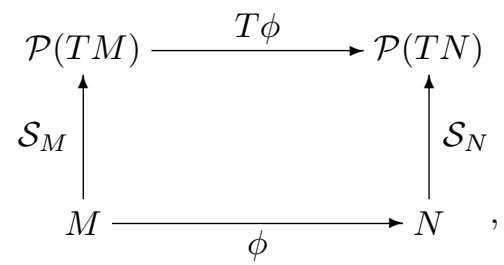

where $\mathcal{P}(T M)$ denotes the powerset of $T_{x} M$ for every $x \in M$.

We see that if $\mathcal{S}_{M}$ is in fact a vector field we recover the notion of projectable vector fields. The notion of projectable control sections is stronger then the notion of Con morphism since for any $x_{1}, x_{2} \in M$ such that $\phi\left(x_{1}\right)=\phi\left(x_{2}\right)$ we necessarily have $T \phi\left(\mathcal{S}_{M}\left(x_{1}\right)\right)=\mathcal{S}_{N} \circ \phi\left(x_{1}\right)=T \phi\left(\mathcal{S}_{M}\left(x_{2}\right)\right)$ if $\mathcal{S}_{M}$ is projectable. On the other hand, if $(\phi, \varphi)$ is a Con morphism for a fiber preserving lift $\varphi$ of $\phi$, we only have the inclusions $T \phi\left(\mathcal{S}_{M}\left(x_{1}\right)\right) \subseteq \mathcal{S}_{N} \circ \phi\left(x_{1}\right)$ and $T \phi\left(\mathcal{S}_{M}\left(x_{2}\right)\right) \subseteq \mathcal{S}_{N} \circ \phi\left(x_{1}\right)$. Therefore, projectability 
with respect to $\phi$ and AII implies that $\phi$ can be extended to a Con morphism but given a Con morphism $f=(\phi, \varphi)$ from $\Sigma_{M}$ to $\Sigma_{N}$ it is not true, in general, that $\mathcal{S}_{M}$ is projectable with respect to $\phi$.

To determine the relevant conditions on $\mathcal{S}_{M}$ that ensure projectability we will need an auxiliary result.

Lemma 6.2. Let $f: M \rightarrow N$ be a map between manifolds and let $X_{t}$ be the flow of a vector field $X \in T M$ such that $f \circ X_{t}=f$. Then the following equality holds for every $x \in M$ :

$$
T_{x} f T_{X_{t}(x)} X_{-t}=T_{X_{t}(x)} f
$$

Proof. The equality $f \circ X_{t}=f$ is equivalent to

$$
\begin{aligned}
& f \circ X_{t}(x)=f(x) \\
\Leftrightarrow & f\left(X_{t}(x)\right)=f \circ\left(X_{t}\right)^{-1} \circ X_{t}(x) \\
\Leftrightarrow & f\left(X_{t}(x)\right)=f \circ X_{-t}\left(X_{t}(x)\right),
\end{aligned}
$$

and by differentiation of the previous expression we arrive at the desired equality

$$
T_{X_{t}(x)} f=T_{x} f T_{X_{t}(x)} X_{-t} .
$$

We can now give sufficient and necessary conditions for projectability of control sections.

Proposition 6.3 (projectable control sections). Let $M$ be a manifold, $\mathcal{S}_{M}$ a control section on $M$, and $\phi: M \rightarrow N$ a surjective submersion with connected fibers. Given any control system $\left(U_{M}, F_{M}\right)$ satisfying $\mathbf{A I}$ and defining $\mathcal{S}_{M}$, and any $\widehat{F_{M}} \in$ $F_{M}^{e}, \mathcal{S}_{M}$ is projectable with respect to $\phi$ iff

$$
\left[\widehat{F_{M}}, \operatorname{ker}\left(T \pi_{U_{M}}^{*} \phi\right)\right] \subseteq \operatorname{ker}\left(T \pi_{U_{M}}^{*} \phi\right)+\left[\widehat{F_{M}}, \operatorname{ker}\left(T \pi_{U_{M}}\right)\right]
$$

Proof. We show necessity first. Assume that diagram (6.1) commutes. Then we have

$$
T_{x} \phi\left(\mathcal{S}_{M}(x)\right)=T_{x^{\prime}} \phi\left(\mathcal{S}_{M}\left(x^{\prime}\right)\right)
$$

for all $x, x^{\prime} \in M$ such that $\phi(x)=\phi\left(x^{\prime}\right)$, that is, for any $x$ and $x^{\prime}$ on the same leaf of the foliation induced by $\operatorname{ker}(T \phi)$. If we denote by $K_{t}$ the flow of any vector field $K \in \operatorname{ker}\left(T \pi_{U_{M}}^{*} \phi\right)$, expression (6.6) implies that

$$
T_{\pi_{U_{M}} \circ K_{t}(u)} \phi\left(F_{M} \circ K_{t}(u)\right) \in T_{x} \phi\left(\mathcal{S}_{M}(x)\right)
$$

for every $t \in \mathbb{R}$ such that $K_{t}$ is defined and for every $u \in \pi_{U_{M}}^{-1}(x)$. Since the left-hand side of (6.7) belongs to the right-hand side and $\pi_{U_{M}}^{-1}(x)$ is connected by AI, we can always find a $Y \in \operatorname{ker}\left(T \pi_{U_{M}}\right)$ such that its flow $Y_{t}$ will parameterize the image of the left-hand side. That is

$$
T_{\pi_{U_{M}} \circ K_{t}(u)} \phi\left(F_{M} \circ K_{t}(u)\right)=T_{\pi_{U_{M}} \circ Y_{t}(u)} \phi\left(F_{M} \circ Y_{t}(u)\right) .
$$

The previous equality implies that for any $\widehat{F_{M}} \in F_{M}^{e}$ we have

$$
T_{K_{t}(u)} \pi_{U_{M}}^{*} \phi\left(\widehat{F_{M}} \circ K_{t}(u)\right)=T_{Y_{t}(u)} \pi_{U_{M}}^{*} \phi\left(\widehat{F_{M}} \circ Y_{t}(u)\right) ;
$$


however, the equalities $\pi_{U_{M}}^{*} \phi \circ K_{t}=K_{t}, \pi_{U_{M}}^{*} \phi \circ Y_{t}=Y_{t}$ and Lemma 6.2 allow one to rewrite (6.9) as

$$
\begin{aligned}
T_{u} \pi_{U_{M}}^{*} \phi\left(T_{K_{t}(u)} K_{-t} \circ \widehat{F_{M}} \circ K_{t}(u)\right) & =T_{u} \pi_{U_{M}}^{*} \phi\left(T_{Y_{t}(u)} Y_{-t} \circ \widehat{F_{M}} \circ Y_{t}(u)\right) \\
\Leftrightarrow \quad T_{u} \pi_{U_{M}}^{*} \phi\left(K_{t}(u)^{*} \widehat{F_{M}}\right) & =T_{u} \pi_{U_{M}}^{*} \phi\left(Y_{t}(u)^{*} \widehat{F_{M}}\right) .
\end{aligned}
$$

Time differentiation at $t=0$ now implies

$$
\begin{aligned}
T_{u} \pi_{U_{M}}^{*} \phi\left(\left[K(u), \widehat{F_{M}}(u)\right]\right) & =T_{u} \pi_{U_{M}}^{*} \phi\left(\left[Y(u), \widehat{F_{M}}(u)\right]\right) \\
\Rightarrow\left[K, \widehat{F_{M}}\right] & \in\left[Y, \widehat{F_{M}}\right]+\operatorname{ker}\left(T \pi_{U_{M}}^{*} \phi\right),
\end{aligned}
$$

which trivially implies inclusion (6.5).

To show sufficiency we use a similar argument. Assume that (6.5) holds; then for any $K \in \operatorname{ker}\left(T \pi_{U_{M}}^{*} \phi\right)$ there exists a $Y \in \operatorname{ker}\left(T \pi_{U_{M}}\right)$ such that

$$
\begin{aligned}
& \left.\left.T_{u} \pi_{U_{M}}^{*} \phi\left(\widehat{F_{M}}(u), K(u)\right]\right)=T_{u} \pi_{U_{M}}^{*} \phi\left(\widehat{F_{M}}(u), Y(u)\right]\right) \\
\Leftrightarrow & T_{u} \pi_{U_{M}}^{*} \phi\left(\left[\widehat{F_{M}}(u), K(u)-Y(u)\right]\right)=0 .
\end{aligned}
$$

Consider now the regular and involutive distribution $\operatorname{ker}\left(T \pi_{U_{M}}^{*} \phi\right)$. Involutivity and regularity imply that $Z_{t}^{*} W \in \operatorname{ker}\left(T \pi_{U_{M}}^{*} \phi\right)$ for any $W \in \operatorname{ker}\left(T \pi_{U_{M}}^{*} \phi\right)$ and the flow $Z_{t}$ of any vector field $Z \in \operatorname{ker}\left(T \pi_{U_{M}}^{*} \phi\right)$ [34]. Since $K \in \operatorname{ker}\left(T \pi_{U_{M}}^{*} \phi\right)$ and $Y \in \operatorname{ker}\left(T \pi_{U_{M}}^{*} \phi\right)$, it follows that $K-Y \in \operatorname{ker}\left(T \pi_{U_{M}}^{*} \phi\right)$, but from (6.12), $\left[\widehat{F_{M}}, K-Y\right]$ also belongs to $\operatorname{ker}\left(T \pi_{U_{M}}^{*} \phi\right)$ so that we conclude

$$
T_{u} \pi_{U_{M}}^{*} \phi\left((K-Y)_{t}(u)^{*}\left[\widehat{F_{M}}, K-Y\right]\right)=0,
$$

where $(K-Y)_{t}$ denotes the flow of the vector field $K-Y$. However, the previous expression is equivalent to

$$
\begin{aligned}
& T_{u} \pi_{U_{M}}^{*} \phi\left(\frac{d}{d t}(K-Y)_{t}(u)^{*} \widehat{F_{M}}\right)=0 \\
\Leftrightarrow & \frac{d}{d t} T_{u} \pi_{U_{M}}^{*} \phi\left((K-Y)_{t}(u)^{*} \widehat{F_{M}}\right)=0,
\end{aligned}
$$

where the last equality follows from the fact that $T \phi$ is a linear map. Since the time derivative is zero, we must have

$$
T_{u} \pi_{U_{M}}^{*} \phi\left((K-Y)_{t}(u)^{*} \widehat{F_{M}}\right)=T_{u} \pi_{U_{M}}^{*} \phi\left((K-Y)_{0}(u)^{*} \widehat{F_{M}}\right)=T_{u} \pi_{U_{M}}^{*} \phi\left(\widehat{F_{M}}(u)\right) .
$$

From the equality $\pi_{U_{M}}^{*} \phi=\pi_{U_{M}}^{*} \phi \circ(K-Y)_{t}$ we conclude that $T_{u} \pi_{U_{M}}^{*} \phi T_{(K-Y)_{t}(u)}(K-$ $Y)_{-t}=T_{(K-Y)_{t}(u)} \pi_{U_{M}}^{*} \phi$ by Lemma 6.2 so that $(6.15)$ can be written as

$$
T_{(K-Y)_{t}(u)} \pi_{U_{M}}^{*} \phi\left(\widehat{F_{M}} \circ(K-Y)_{t}(u)\right)=T_{u} \pi_{U_{M}}^{*} \phi\left(\widehat{F_{M}}(u)\right)
$$

and projecting on $T M$ we get

$$
T_{\pi_{U_{M}}\left(K_{t}^{\prime}(u)\right)} \phi\left(F_{M} \circ\left(K^{\prime}\right)_{t}(u)\right)=T_{x} \phi\left(F_{M}(u)\right)
$$


with $K^{\prime}=K-Y$. This equality shows that for any $X \in \mathcal{S}_{M}(x), T_{x} \phi \cdot X \in$ $T_{x^{\prime}} \phi\left(\mathcal{S}_{M}\left(x^{\prime}\right)\right)$; therefore, $T_{x} \phi\left(\mathcal{S}_{M}(x)\right) \subseteq T_{x^{\prime}} \phi\left(\mathcal{S}_{M}\left(x^{\prime}\right)\right)$. However, replacing $x$ by $x^{\prime}$ and $K$ by $-K$ on (6.17), we get $T_{x^{\prime}} \phi\left(\mathcal{S}_{M}\left(x^{\prime}\right)\right) \subseteq T_{x} \phi\left(\mathcal{S}_{M}(x)\right)$ so that we conclude the equality

$$
T_{x} \phi\left(\mathcal{S}_{M}(x)\right)=T_{x^{\prime}} \phi\left(\mathcal{S}_{M}\left(x^{\prime}\right)\right) .
$$

By connectedness of the fibers $\phi^{-1}(y)$ any point $x^{\prime \prime}$ satisfying $\phi\left(x^{\prime \prime}\right)=\phi(x)$ can be reached by a concatenation of flows induced by vector fields in $\operatorname{ker}(T \phi)$. Transitivity of equality between sets implies that (6.18) holds for any two points $x, x^{\prime} \in M$ such that $\phi(x)=\phi\left(x^{\prime}\right)$ from which commutativity of diagram (6.1) readily follows.

By now it is already clear that projectability and local controlled invariance are equivalent concepts. We recall the notion of locally controlled invariant distribution.

Definition 6.4 (locally controlled invariant distributions [27]). Let $\Sigma_{M}=$ $\left(U_{M}, F_{M}\right)$ be a control system on manifold $M$ and let $\mathcal{D}$ be a distribution on $M$. Distribution $\mathcal{D}$ is locally controlled invariant for $F_{M}$ if for every $x \in M$ there exist an open set $O \subseteq M$ containing $x$ and a local (feedback) isomorphism over the identity $\alpha$ such that in adapted coordinates $(x, v)$ the new control system $F_{M} \circ \alpha$ satisfies

$$
\left[F_{M} \circ \alpha(x, v), \mathcal{D}(x)\right] \subseteq \mathcal{D}(x)
$$

for every $(x, v)$ in the domain of $\alpha$.

If a control section is projectable, then locally we can always choose $\widehat{F_{M}}=F_{M}^{l}$ and therefore recover the conditions for local controlled invariance from [8].

TheOREM 6.5 (see [8]). Let $\Sigma_{M}$ be a control system on manifold $M$ satisfying AI and $\phi: M \longrightarrow N$ a surjective submersion with connected fibers. The distribution $\operatorname{ker}(T \phi)$ is locally controlled invariant for $F_{M}$ iff $\mathcal{S}_{M}$ is projectable with respect to $\phi$.

Even though controlled invariance and projectability are equivalent concepts, we shall use the notion of projectability to describe control sections that behave well under projection instead of controlled invariance which was introduced to describe certain control enforced invariance properties of control systems [42].

From the study of symmetries of nonlinear control systems $[9,26]$ it was already known that the existence of symmetries or partial symmetries implies controlled invariance of a certain distribution associated with the symmetries. This shows that control systems that are projectable comprise quotients by symmetry and controlled invariance. Furthermore, quotients induced by indistinguishability, as discussed in [35], are also of this type. However, there are also quotients for which projectability does not hold as we describe in the next section. Furthermore, as the existence of symmetries can be considered a rare phenomena [32], it is especially important to understand the structure of general nonprojectable quotients.

7. The structure of quotient control systems. We have already seen that the notion of Con morphisms generalizes the notion of projectable control sections. This shows that it is possible to quotient control systems whose control sections are nonprojectable. In this situation the map $\varphi$ and the input space of the quotient control system will be significantly different from the projectable case. To understand this difference we start characterizing the fiber preserving lift $\varphi$ of $\phi$. Recall that if $f=(\phi, \varphi)$ is a morphism from $\Sigma_{M}$ to $\Sigma_{N}$, we have the following commutative diagram: 


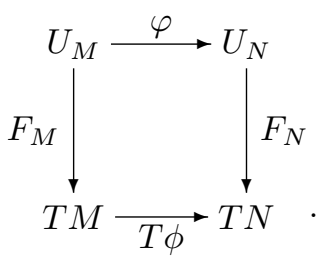

Since $\varphi$ is a surjective submersion, we know that $U_{N}$ is diffeomorphic to $U_{M} / \sim$, where $\sim$ is the regular equivalence relation induced by $\varphi$. This means that to understand the structure of $U_{N}$ it is enough to determine the regular and involutive distribution on $U_{M}$ given by $\operatorname{ker}(T \varphi)$. However, the map $\varphi$ is completely unknown, so we will resort to the elements that are available, namely, $F_{M}$ and $\phi$, to determine $\operatorname{ker}(T \varphi)$. Differentiating ${ }^{5}$ diagram (7.1) we get

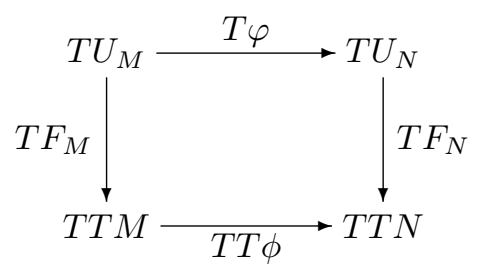

from which we conclude that

$$
\operatorname{ker}\left(T T \phi \circ T F_{M}\right)=\operatorname{ker}\left(T F_{N} \circ T \varphi\right)=\operatorname{ker}(T \varphi),
$$

where the last equality holds since $F_{N}$ is an immersion, provided that assumption AIII holds. We can now attempt to understand what is factored away and what is propagated from $U_{M}$ to $U_{N}$ since $\operatorname{ker}(T \varphi)$ is expressible in terms of $F_{M}$ and $\phi$. The first step is to clarify the relation between $\operatorname{ker}(T \varphi)$ and $\operatorname{ker}(T \phi)$. Since $\varphi$ is a fiber preserving lift of $\phi$, the following diagram commutes:

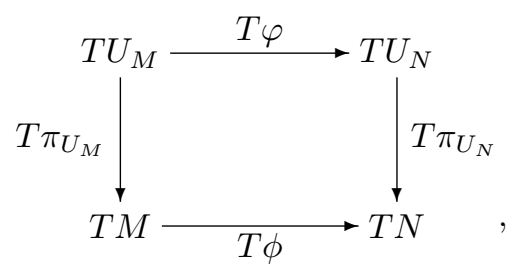

which implies that

$$
T \pi_{U_{M}}(\operatorname{ker}(T \varphi)) \subseteq \operatorname{ker}(T \phi) .
$$

However, this only tells us that the reduction on $M$ due to $\phi$ cannot be "smaller" than the reduction on the base space of $U_{M}$ due to $\varphi$. This leads to the interesting phenomena which occurs when, e.g.,

$$
T \pi_{U_{M}}(\operatorname{ker}(T \varphi))=\{0\} \subseteq \operatorname{ker}(T \phi) .
$$

\footnotetext{
${ }^{5}$ The operator sending manifolds to their tangent manifolds and maps to their tangent maps is an endofunctor on Man, also called the tangent functor [15].
} 
The above expression implies that the base space of $U_{M}$ is not reduced by $\varphi$. However, $U_{N}$ is a fibered manifold with base space $N$ and therefore the points reduced by $\phi$ must necessarily move to the fibers of $U_{N}$. This means that points $u, u^{\prime} \in U_{M}$ satisfying $\pi_{U_{M}}(u) \neq \pi_{U_{M}}\left(u^{\prime}\right)$ will be mapped by $\varphi$ to points satisfying $\pi_{U_{N}} \circ \varphi(u)=\pi_{U_{N}} \circ \varphi\left(u^{\prime}\right)$ and $\varphi(u) \neq \varphi\left(u^{\prime}\right)$. This will not happen if we can ensure the existence of a distribution $\mathcal{D} \subseteq \operatorname{ker}(T \varphi)$ such that $T \pi_{U_{M}}(\mathcal{D})=\operatorname{ker}(T \phi)$. The existence of such a distribution turns out to be related with projectability. To show such a fact we need the following characterization of $\operatorname{ker}(T \varphi)$.

Lemma 7.1. Let $\Sigma_{M}=\left(U_{M}, F_{M}\right)$ be a control system on manifold $M, \phi: M$ $\rightarrow N$ a surjective submersion and $\varphi: U_{M} \longrightarrow U_{N}$ a fiber preserving lift of $\phi$ which is also a submersion, and assume that AIII holds. Under these assumptions, a regular distribution $\mathcal{D} \subseteq T U_{M}$ belongs to $\operatorname{ker}(T \varphi)$ iff

$$
\left.\widehat{\left[F_{M}\right.}, \mathcal{D}\right] ; \subseteq \operatorname{ker}\left(T \pi_{U_{M}}^{*}\right),
$$

where $\widehat{F_{M}}$ is any vector field in $F_{M}^{e}$.

Proof. Assume the existence of the distribution $\mathcal{D}$; then $\mathcal{D} \subseteq \operatorname{ker}(T \varphi)$ is equivalent to

$$
T T \phi \circ T F_{M}(\mathcal{D})=\{0\}
$$

since AIII holds. Let $Z \in \mathcal{D}$ and denote by $Z_{t}$ the flow of $Z$. Expression (7.8) implies that

$$
\left.\frac{d}{d t}\right|_{t=0} T_{\pi_{U_{M}} \circ Z_{t}(u)} \phi\left(F_{M} \circ Z_{t}(u)\right)=\left.0 \Rightarrow \frac{d}{d t}\right|_{t=0} T_{Z_{t}(u)} \pi_{U_{M}}^{*} \phi\left(\widehat{F_{M}} \circ Z_{t}(u)\right)=0
$$

for any $\widehat{F_{M}} \in F_{M}^{e}$ and for all $t \in \mathbb{R}$ such that $Z_{t}$ is defined.

Noticing that $Z \in \mathcal{D} \subseteq \operatorname{ker}(T \varphi)$ implies $\varphi=\varphi \circ Z_{t}$ (since $\varphi$ is constant on the leaves of the foliation induced by $\operatorname{ker}(T \varphi)$ ) and $\pi_{U_{N}} \circ \varphi=\phi \circ \pi_{U_{M}}$ by commutativity of diagram (4.1), we conclude that $\pi_{U_{M}}^{*} \phi$ is also $Z_{t}$ invariant:

$$
\pi_{U_{M}}^{*} \phi \circ Z_{t}=\phi \circ \pi_{U_{M}} \circ Z_{t}=\left(\pi_{U_{N}} \circ \varphi\right) \circ Z_{t}=\pi_{U_{N}} \circ \varphi=\phi \circ \pi_{U_{M}}=\pi_{U_{M}}^{*} \phi .
$$

Lemma 6.2 now ensures that

$$
T_{Z_{t}(u)} \pi_{U_{M}}^{*} \phi=T_{u} \pi_{U_{M}}^{*} \phi \circ T_{Z_{t}(u)} Z_{-t}
$$

and expression (7.11) allows one to rewrite (7.9) as

$$
\begin{aligned}
\left.\frac{d}{d t}\right|_{t=0} T_{Z_{t}(u)} \pi_{U_{M}}^{*} \phi\left(\widehat{F_{M}} \circ Z_{t}(u)\right)=0 & \left.\Leftrightarrow \frac{d}{d t}\right|_{t=0} T_{u} \pi_{U_{M}}^{*} \phi\left(T_{Z_{t}(u)} Z_{-t} \circ \widehat{F_{M}} \circ Z_{t}(u)\right)=0 \\
& \left.\Leftrightarrow \frac{d}{d t}\right|_{t=0} T_{u} \pi_{U_{M}}^{*} \phi\left(Z_{t}(u)^{*} \widehat{F_{M}}\right)=0 \\
& \Leftrightarrow T_{u} \pi_{U_{M}}^{*} \phi\left(\left[Z(u), \widehat{F_{M}}(u)\right]\right)=0
\end{aligned}
$$

or, equivalently, $\left[Z, \widehat{F_{M}}\right] \in \operatorname{ker}\left(T \pi_{U_{M}}^{*} \phi\right)$. Since $Z$ is any vector field in $\mathcal{D}$ it follows that $\left[\widehat{F_{M}}, \mathcal{D}\right] \subseteq \operatorname{ker}\left(T \pi_{U_{M}}^{*} \phi\right)$ as desired.

The converse is similarly proved.

Using Lemma 7.1, we can now characterize the existence of a distribution $\mathcal{D} \subseteq$ $\operatorname{ker}(T \varphi)$ projecting on $\operatorname{ker}(T \phi)$. 
Proposition 7.2. Let $\Sigma_{M}=\left(U_{M}, F_{M}\right)$ be a control system on manifold $M$ satisfying AI, $\phi: M \longrightarrow N$ a surjective submersion, and $\varphi: U_{M} \rightarrow U_{N}$ a fiber preserving lift of $\phi$ which is also a submersion. There exists a regular distribution $\mathcal{D}$ on $U_{M}$ satisfying $\mathcal{D} \subseteq \operatorname{ker}(T \varphi)$ and $T \pi_{U_{M}}(\mathcal{D})=\operatorname{ker}(T \phi)$ iff $\mathcal{S}_{M}$ is projectable with respect to $\phi$.

Proof. We start by showing that projectability implies the existence of $\mathcal{D}$. If $\mathcal{S}_{M}$ is projectable with respect to $\phi$, then for every $x, x^{\prime} \in M$ such that $\phi(x)=\phi\left(x^{\prime}\right)$ we have that $T_{x} \phi\left(\mathcal{S}_{M}(x)\right)=T_{x^{\prime}} \phi\left(\mathcal{S}_{M}\left(x^{\prime}\right)\right)$. This means that for any $x \in M, u \in \pi_{U_{M}}^{-1}(x)$, and $X \in \operatorname{ker}\left(T \pi_{U_{M}}^{*} \phi\right)$, there exists a $Y \in \operatorname{ker}\left(T \pi_{U_{M}}\right)\left(\right.$ recall that $\pi_{U_{M}}^{-1}(x)$ is connected by $\mathbf{A I}$ ) such that

$$
T_{\pi_{U_{M}} \circ X_{t}(u)} \phi\left(F_{M} \circ X_{t}(u)\right)=T_{x} \phi\left(F_{M} \circ Y_{t}(u)\right)
$$

for all $t \in \mathbb{R}$ such that the flows $X_{t}$ and $Y_{t}$ of $X$ and $Y$ are defined. Considering now $T \phi$ as a map between the manifolds $T M$ and $T N$, the time derivative of $T_{\alpha(t)} \phi(\beta(t))$ for $(\alpha, \beta): \mathbb{R} \rightarrow T M$ provides $T_{(\alpha(t), \beta(t))} T_{\alpha(t)} \phi(T \beta(t))$. The same considerations applied to (7.13) at $t=0$ give

$$
T_{\left(x, F_{M}(u)\right)} T_{x} \phi \circ T_{u} F_{M}(X(u))=T_{\left(x, F_{M}(u)\right)} T_{x} \phi \circ T_{u} F_{M}(Y(u)),
$$

which we rewrite as

$$
T_{\left(x, F_{M}(u)\right)} T_{x} \phi \circ T_{u} F_{M}(X(u)-Y(u))=0
$$

by linearity of the involved maps. Since (7.15) is true for any $X \in \operatorname{ker}\left(T \pi_{U_{M}}^{*} \phi\right)$, we can define the distribution

$\mathcal{D}=\bigcup_{K \in \operatorname{ker}(T \phi)}\left\{Z=X-Y: X \in K^{e} \wedge Y \in \operatorname{ker}\left(T \pi_{U_{M}}\right)\right.$ is such that (7.15) holds $\}$.

This distribution clearly satisfies

$$
T T \phi \circ T F_{M}(\mathcal{D})=\{0\} \quad \Leftrightarrow \quad \mathcal{D} \subseteq \operatorname{ker}(T \varphi),
$$

is regular since $\operatorname{dim}(\mathcal{D})=\operatorname{dim}(\operatorname{ker}(T \phi))$ by construction, satisfies $T \pi_{U_{M}}(\mathcal{D})=\operatorname{ker}(T \phi)$ also by construction, and is therefore the desired distribution.

The converse is proved as follows. Assume the existence of the distribution $\mathcal{D} \subseteq$ $\operatorname{ker}(T \varphi)$. By Lemma 7.1 we have

$$
\left.\widehat{\left[F_{M}\right.}, \mathcal{D}\right] \subseteq \operatorname{ker}\left(T \pi_{U_{M}}^{*}\right)
$$

Since $T \pi_{U_{M}}(\mathcal{D})=\operatorname{ker}(T \phi)$ it follows that $\mathcal{D}+T \pi_{U_{M}}=T \pi_{U_{M}}^{*} \phi$ and

$$
\begin{aligned}
& {\left[\widehat{F_{M}}, \mathcal{D}\right] \subseteq \operatorname{ker}\left(T \pi_{U_{M}}^{*}\right) } \\
\Rightarrow & {\left[\widehat{F_{M}}, \mathcal{D}+\operatorname{ker}\left(T \pi_{U_{M}}\right)\right] \subseteq \operatorname{ker}\left(T \pi_{U_{M}}^{*}\right)+\left[\widehat{F_{M}}, \operatorname{ker}\left(T \pi_{U_{M}}\right)\right] } \\
\Rightarrow & {\left[\widehat{F_{M}}, \operatorname{ker}\left(T \pi_{U_{M}}^{*} \phi\right)\right] \subseteq \operatorname{ker}\left(T \pi_{U_{M}}^{*}\right)+\left[\widehat{F_{M}}, \operatorname{ker}\left(T \pi_{U_{M}}\right)\right], }
\end{aligned}
$$

which combined with Proposition 6.3 shows that $\mathcal{S}_{M}$ is projectable with respect to $\phi$ as desired. 
Collecting the results given by Lemma 7.1 and Proposition 7.2 we can now characterize both $\varphi$ and $U_{N}$. Intuitively, we will use projectability to determine if the fibers of the quotient control system will receive states from $M$ and Lemma 7.1 to characterize the amount of reduction induced by $\varphi$ on the fibers of $\pi_{U_{M}}$.

THEOREM 7.3 (structure of control systems quotients). Consider a control system $\Sigma_{M}=\left(U_{M}, F_{M}\right)$ over a manifold $M$ satisfying $\mathbf{A I}$, let $\left(f, \Sigma_{N}\right)=\left((\phi, \varphi),\left(U_{N}, F_{N}\right)\right)$ be a quotient of $\Sigma_{M}$ where $\phi$ has connected fibers, and assume that AIII holds. Let $\mathcal{E}$ be the involutive distribution defined by $\mathcal{E}=\left\{X \in \operatorname{ker}\left(T \pi_{U_{M}}\right):\left[\widehat{F_{M}}, X\right] \in \operatorname{ker}\left(T \pi_{U_{M}}^{*} \phi\right)\right\}$, which we assume to be regular, denote by $R_{\mathcal{E}}$ the regular equivalence relation induced by $\mathcal{E}$, and let $\widehat{F_{M}}$ be any vector field in $F_{M}^{e}$. Under these assumptions the following can be stated:

1. Reduction from states to states and no reduction on inputs. Fibered manifold $U_{N}$ has base space diffeomorphic to $N$, and fibers $\pi_{U_{N}}^{-1}(y)$ diffeomorphic to $\pi_{U_{M}}^{-1}(x)$, $\phi(x)=y$ iff

(i) $\mathcal{S}_{M}$ is projectable with respect to $\phi$;

(ii) $\mathcal{E}=\{0\}$.

2. Reduction from states to states and from inputs to inputs. Fibered manifold $U_{N}$ has base space diffeomorphic to $N$, and fibers $\pi_{U_{N}}^{-1}(y)$ diffeomorphic to $\pi_{U_{M}}^{-1}(x) / R_{\mathcal{E}}$, $\phi(x)=y$ iff

(i) $\mathcal{S}_{M}$ is projectable with respect to $\phi$;

(ii) $\mathcal{E} \neq\{0\}$.

3. Reduction from states to inputs and no reduction on inputs. Fibered manifold $U_{N}$ has base space diffeomorphic to $N$, and fibers $\pi_{U_{N}}^{-1}(y)$ diffeomorphic to $\pi_{U_{M}}^{-1}\left(\phi^{-1}(y)\right), \phi(x)=y$ iff

(i) for all $K \in \operatorname{ker}\left(T \pi_{U_{M}}^{*} \phi\right), \quad\left[\widehat{F_{M}}, K\right] \notin \operatorname{ker}\left(T \pi_{U_{M}}^{*} \phi\right)$;

(ii) $\mathcal{E}=\{0\}$.

4. Reduction from states to inputs and from inputs to inputs. Fibered manifold $U_{N}$ has base space diffeomorphic to $N$, and fibers $\pi_{U_{N}}^{-1}(y)$ diffeomorphic to $\left(\pi_{U_{M}}^{-1} / R_{\mathcal{E}}\right)\left(\phi^{-1}(y)\right), \phi(x)=y$ iff

(i) for all $K \in \operatorname{ker}\left(T \pi_{U_{M}}^{*} \phi\right), \quad\left[\widehat{F_{M}}, K\right] \notin \operatorname{ker}\left(T \pi_{U_{M}}^{*} \phi\right)$;

(ii) $\mathcal{E} \neq\{0\}$.

Proof. We note that in all four cases the base space of $U_{N}$ is diffeomorphic to $N$, since $U_{N}$ is equipped with a surjective submersion $\pi_{U_{N}}: U_{N} \rightarrow N$. We will, therefore, only discuss the characterization of fibers of $\pi_{U_{N}}$. We follow the enumeration of the theorem.

1 and 2: Since $\varphi$ is fiber preserving, we denote by $\varphi_{x}: \pi_{U_{M}}^{-1}(x) \rightarrow \pi_{U_{N}}^{-1}(\phi(x))$ the restriction of $\varphi$ to the fibers $\pi_{U_{M}}^{-1}(x), x \in M$. We now claim that projectability implies $\varphi_{x}\left(\pi_{U_{M}}^{-1}(x)\right)=\varphi_{x^{\prime}}\left(\pi_{U_{M}}^{-1}\left(x^{\prime}\right)\right)$ for every $x, x^{\prime} \in M$ such that $\phi(x)=\phi\left(x^{\prime}\right)$. Recall that by definition of projectability, we have $T_{x} \phi\left(\mathcal{S}_{M}(x)\right)=T_{x^{\prime}} \phi\left(\mathcal{S}_{M}\left(x^{\prime}\right)\right)$. However, $\mathcal{S}_{M}(x)=F_{M}\left(\pi_{U_{M}}^{-1}(x)\right)$ so that we conclude $T_{x} \phi \circ F_{M}\left(\pi_{U_{M}}^{-1}(x)\right)=T_{x^{\prime}} \phi \circ F_{M}\left(\pi_{U_{M}}^{-1}\left(x^{\prime}\right)\right)$. From assumption AIII follows injectivity of $F_{N}$, which combined with commutativity of the second diagram in (4.1) leads to $\varphi_{x}\left(\pi_{M}^{-1}(x)\right)=\varphi_{x^{\prime}}\left(\pi_{M}^{-1}\left(x^{\prime}\right)\right)$, as desired. This equality also shows that $\varphi_{x}$ is surjective since $\varphi$ is. Furthermore, we conclude that to characterize $\pi_{U_{N}}^{-1}(y)$ it suffices to characterize the image of $\varphi_{x}$ for some $x \in \phi^{-1}(y)$. We now consider $\operatorname{ker}(T \varphi(x)) \cap \operatorname{ker}\left(T \pi_{U_{M}}^{-1}(x)\right)$, which by Lemma 7.1 is equal to $\mathcal{E}$ and is regular by assumption. Fields in $\pi_{U_{M}}^{-1}(x)$ and the equivalence relation $R_{\mathcal{E}}$ can be identified with an equivalence relation on $\pi_{U_{M}}^{-1}(x)$. We first note that projectability implies via Proposition 7.2 and (7.5) that $T \pi_{U_{M}}(\operatorname{ker}(T \varphi))=\operatorname{ker}(T \phi)$. This shows 
that

$$
\operatorname{dim}(\operatorname{ker}(T \varphi))=\operatorname{dim}(\operatorname{ker}(T \phi))+\operatorname{dim}(\mathcal{E})
$$

On the other hand,

$$
\begin{aligned}
\operatorname{dim}\left(\pi_{U_{N}}^{-1}(y)\right) & =\operatorname{dim}\left(U_{N}\right)-\operatorname{dim}(N) \\
& =\operatorname{dim}\left(U_{M}\right)-\operatorname{dim}(\operatorname{ker}(T \varphi))-\operatorname{dim}(N) \\
& =\operatorname{dim}\left(U_{M}\right)-\operatorname{dim}(\operatorname{ker}(T \phi))-\operatorname{dim}(\mathcal{E})-\operatorname{dim}(N) \quad \text { by }(7.18) \\
& =\operatorname{dim}\left(U_{M}\right)-\operatorname{dim}(\operatorname{ker}(T \phi))-\operatorname{dim}(\mathcal{E})-\operatorname{dim}(M)+\operatorname{dim}(\operatorname{ker}(T \phi)) \\
& =\operatorname{dim}\left(U_{M}\right)-\operatorname{dim}(\mathcal{E})-\operatorname{dim}(M) \\
& =\operatorname{dim}\left(\pi_{U_{M}}^{-1}(x)\right)-\operatorname{dim}(\mathcal{E})=\operatorname{rank}\left(\varphi_{x}\right),
\end{aligned}
$$

which shows that $\varphi_{x}$ is a submersion. We thus see that $\pi_{U_{N}}^{-1}(y)$ can now be identified with $\pi_{U_{M}}^{-1}(x) / R_{\mathcal{E}}$ since every vector field $X \in \mathcal{E}$ satisfies $T \pi_{U_{M}}(X)=0$ and therefore induces a vector field on $\pi_{U_{M}}^{-1}(x)$. If $\mathcal{E}=\{0\}$, it follows that $\pi_{U_{N}}^{-1}(y) \cong \pi_{U_{M}}^{-1}(x) / R_{\mathcal{E}} \cong$ $\pi_{U_{M}}^{-1}(x)$ as required by case 1 .

Conversely, since the base of $U_{N}$ is diffeomorphic to the quotient of $M$ by the regular equivalence relation induced by $\operatorname{ker}(T \phi)$ and the fibers of $\pi_{U_{N}}$ diffeomorphic to $\pi_{U_{M}} / R_{\mathcal{E}}$, it follows that $\operatorname{ker}(T \varphi)$ can be locally described by $\mathcal{D} \oplus \mathcal{E}$ for $\mathcal{D}=$ $\operatorname{ker}(T \phi)^{l}$ and $T \pi_{U_{M}}(\mathcal{E})=\{0\}$. From the existence of $\mathcal{D}$ and Proposition 6.3 follows projectability of $\mathcal{S}_{M}(x)$. Furthermore, if the fibers of $\pi_{U_{M}}$ are diffeomorphic to the fibers of $\pi_{U_{N}}$, we have $\mathcal{E}=\{0\}$ (case 1) and otherwise $\mathcal{E} \neq\{0\}$ (case 2).

3 and 4: From assumption (i) and Lemma 7.1 we conclude that there exists no $X \neq 0$ belonging to $K(T \varphi)$ such that $T \pi_{U_{M}}(X) \in \operatorname{ker}(T \phi)$. Since $T \pi_{U_{M}}(\operatorname{ker}(T \varphi)) \subseteq$ $\operatorname{ker}(T \phi)$ (see the discussion before $(7.5))$, it follows that $T \pi_{U_{M}}(\operatorname{ker}(T \varphi))=\{0\}$. Consequently, every $X \in \operatorname{ker}(T \varphi)$ is tangent to $\pi_{U_{M}}^{-1}(x)$ and $\varphi\left(U_{M}\right)$ is diffeomorphic to a fibered manifold with base space $M$ and fibers $\pi_{U_{M}}^{-1}(x) / R_{\mathcal{E}}$. Let us denote by $\pi: \varphi\left(U_{M}\right) \rightarrow M$ the projection from total space to base space which clearly satisfies $\pi_{U_{M}}=\pi \circ \varphi$. We now use the fact $\pi_{U_{N}} \circ \varphi=\phi \circ \pi_{U_{M}}$ with $\pi_{U_{M}}=\pi \circ \varphi$ to get $\pi_{U_{N}} \circ \varphi=\phi \circ \pi \circ \varphi$ and by surjectivity of $\varphi$ we finally conclude the equality $\pi_{U_{N}}=\phi \circ \pi$. It is now clear that $\pi_{U_{N}}^{-1}(y) \cong \pi^{-1}\left(\phi^{-1}(y)\right) \cong\left(\pi_{U_{M}}^{-1} / R_{\mathcal{E}}\right)\left(\phi^{-1}(y)\right)$ as required by case 4 . Case 3 is obtained by setting $\mathcal{E}=\{0\}$ and obtaining $\pi_{U_{N}}^{-1}(y) \cong$ $\left(\pi_{U_{M}}^{-1} / R_{\mathcal{E}}\right)\left(\phi^{-1}(y)\right) \cong \pi_{U_{M}}^{-1}\left(\phi^{-1}(y)\right)$.

The converse is proved as follows. Since the fibers of $\pi_{U_{N}}$ are diffeomorphic to $\left(\pi_{U_{M}}^{-1} / R_{\mathcal{E}}\right)\left(\phi^{-1}(y)\right)$, we see that points $u, u^{\prime} \in U_{M}$ satisfying $\pi_{U_{M}}(u) \neq \pi_{U_{M}}\left(u^{\prime}\right)$ and $\phi \circ \pi_{U_{M}}(u)=\phi \circ \pi_{U_{M}}\left(u^{\prime}\right)$ also satisfy $\varphi(u) \neq \varphi\left(u^{\prime}\right)$. This shows that no vector field $X \neq 0$ in $\operatorname{ker}\left(T \pi_{U_{M}}^{*} \phi\right)$ belongs to $\operatorname{ker}(T \varphi)$ since otherwise different points in a trajectory of $X$ would violate the above remark. The nonexistence of such vectors $X$ implies, via Lemma 7.1, condition (i) and also implies that $\operatorname{ker}(T \varphi)=\mathcal{E}$. It then follows that if $\pi_{U_{N}}^{-1}(y) \cong \pi_{U_{M}}^{-1}\left(\phi^{-1}(y)\right)$, then $\mathcal{E}=0$ (case 3 ) and $\mathcal{E} \neq 0$ otherwise (case $4)$.

We see that the notion of projectability is fundamentally related to the structure of quotient control systems. If the controlled section $\mathcal{S}_{M}$ is projectable, then the inputs of the quotient control system are the same or a quotient of the original inputs. Projectability can therefore be seen as a structural property of a control system in the sense that it admits special decompositions [11, 27]. However, for general systems not admitting this special structure, that is, for systems that are not projectable, it is still 
possible to construct quotients by moving the neglected state information to the fibers. The states of the original system that are factored out by $\phi$ are regarded as control inputs in the quotient control system. This shows that from a hierarchical synthesis point of view, control systems that are not projectable are much more appealing since one can design control laws for the abstracted system that when pulled-down to the original one are regarded as specifications for the dynamics on the neglected states [37].

8. Conclusions. In this paper quotients of fully nonlinear control systems were investigated. We showed that under mild conditions quotients exist, and we characterized the structure of the quotient state/input space. This was achieved by introducing the category of control systems which was the natural framework to discuss quotients of control systems. One of the important ingredients of the characterization of quotients was the notion of a projectable control section, which being equivalent to controlled invariance allowed one to understand the difference between general quotients and those induced by symmetries, partial symmetries, or controlled invariance.

There are still innumerous directions to be explored. The correct relations of the results presented in this work with the notion of extended control system [25] are not yet understood. This seems to lead to a possible generalization of the constructive procedures presented in [29] to compute quotients of nonlinear control affine systems to fully nonlinear control systems. Other directions being currently investigated include similar results for mechanical control systems where the Hamiltonian structure is preserved by the factorization process [36] as well as hybrid control systems [38].

Acknowledgments. The authors would like to acknowledge the innumerous enlightening discussions with Gonçalo Tabuada, Esfandiar Hagverdi, and Slobodan Simic on categorical interpretations of dynamical and control systems. Equally appreciated were the constructive comments provided by the anonymous reviewers.

\section{REFERENCES}

[1] R. Abraham, J. Marsden, and T. Ratiu, Manifolds, Tensor Analysis, and Applications, 2nd ed., Applied Mathematical Sciences 75, Springer-Verlag, New York, 1988.

[2] M. Arbib And E. G. Manes, Machines in a category: An expository introduction, SIAM Rev., 16 (1974), pp. 163-192.

[3] F. Borceux, Handbook of Categorical Algebra, Cambridge University Press, Cambridge, UK, 1994.

[4] R. Brockett, Control theory and analytical mechanics, in Geometric Control Theory, C. Martin and R. Hermann, eds., Lie Groups: History, Frontiers and Appl. Vol. VII, Math Sci Press, Brookline, MA, 1977, pp. 1-48.

[5] R. Brockett, Feedback invariants for nonlinear systems, in Preprints of the 6th IFAC Congress, Helsinki, Finland, 1978, pp. 1115-1120.

[6] M. Fliess AND I. KUPKA, A finiteness criterion for nonlinear input-output differential systems, SIAM J. Control Optim., 21 (1983), pp. 721-728.

[7] M. Fliess, J. Levine, P. Martin, And P. Rouchon, Flatness and defect of non-linear systems: Introductory theory and examples, Internat. J. Control, 61 (1995), pp. 1327-1361.

[8] K. Grasse, On controlled invariance for fully nonlinear control systems, Internat. J. Control, 56 (1992), pp. 1121-1137.

[9] J. W. GRizzLe AND S. I. Marcus, The structure of nonlinear control systems possessing symmetries, IEEE Trans. Automat. Control, 30 (1985), pp. 248-258.

[10] L. Hunt, R. Su, And G. Meyer, Design for multi-input nonlinear systems, in Differential Geometric Control Theory, R. Brocket, R. Millman, and H. J. Sussmann, eds., Birkhäuser Boston, Boston, 1983, pp. 268-298.

[11] A. Isidori, Nonlinear Control Systems, 3rd ed., Springer-Verlag, Berlin, 1996.

[12] A. Isidori, A. Krener, C. G. Giorgi, and S. Monaco, Nonlinear decoupling via feedback: A differential geometrical approach, IEEE Trans. Automat. Control, 26 (1981), pp. 331-345.

[13] B. Jakubczyk and W. Respondek, On linearization of control systems, Bull. Acad. Polon. Sci. Ser. Sci. Math., 28 (1980), pp. 517-522. 
[14] V. Jurdjevic, Geometric Control Theory, Cambridge Studies in Advanced Mathematics 52, Cambridge University Press, Cambridge, UK, 1997.

[15] I. Kolar, P. W. Michor, and J. SlovaK, Natural Operations in Differential Geometry, Springer-Verlag, Berlin, 1993.

[16] A. Krener, On the equivalence of control systems and linearization of nonlinear systems, SIAM J. Control, 11 (1973), pp. 670-676.

[17] A. J. Krener, A decomposition theory for differentiable systems, SIAM J. Control Optim., 15 (1977), pp. 813-829.

[18] S. MacLane, Categories for the Working Mathematician, Springer-Verlag, New York, 1971.

[19] A. D. LEwIS, The category of affine connection control systems, in Proceedings of the 39th IEEE Conference on Decision and Control, Sydney, Australia, 2000, pp. 1260-1265.

[20] R. Marino, On the largest feedback linearizable subsystem, Systems Control Lett., 6 (1986), pp. $345-351$.

[21] G. Marmo, A. Simoni, B. Vitale, and E. J. Saletan, Dynamical Systems, John Wiley \& Sons, Chichester, UK, 1985.

[22] L. Martin and P. Crouch, Controllability on principal fiber bundles with compact structure group, Syst. Control Lett., 5 (1984), pp. 35-40.

[23] H. NiJMEIJER, Controlled invariance for affine control systems, Internat. J. Control, 34 (1981), pp. $825-833$.

[24] H. NiJMeIJER, Feedback decomposition of nonlinear control systems, IEEE Trans. Automat. Control, 28 (1983), pp. 861-862.

[25] H. NiJMeiJer AND A. VAN DER Schaft, Controlled invariance for nonlinear systems, IEEE Trans. Automat. Control, 27 (1982), pp. 904-914.

[26] H. NiJmeijer and A. VAn Der Schaft, Partial symmetries for nonlinear systems, Math. Systems Theory, 18 (1985), pp. 79-96.

[27] H. Nijmeijer and A. van der Schaft, Nonlinear Dynamical Control Systems, Springer-Verlag, New York, 1990.

[28] G. J. Pappas, G. Lafferriere, and S. Sastry, Hierarchically consistent control systems, IEEE Trans. Automat. Control, 45 (2000), pp. 1144-1160.

[29] G. J. Pappas And S. Simic, Consistent abstractions of affine control systems, IEEE Trans. Automat. Control, 47 (2002), pp. 745-756.

[30] J. W. Polderman and J. Willems, Introduction to Mathematical Systems Theory: A Behavioral Approach, Springer-Verlag, New York, 1998.

[31] W. Respondek, On decomposition of nonlinear control systems, Systems Control Lett., 1 (1981), pp. 301-308.

[32] W. Respondek AND I. A. TAlL, Nonlinearizable single-input control systems do not admit stationary symmetries, Systems Control Lett., 46 (2002), pp. 1-16.

[33] J. Rutten, Universal coalgebra: A theory of systems, Theoret. Comput. Sci., 249 (2000), pp. 3-80.

[34] H. J. Sussmann, Orbits of families of vector fields and integrability of distributions, Trans. Amer. Math. Soc., 180 (1973), pp. 171-188.

[35] H. J. Sussmann, Existence and uniqueness of minimal realizations of nonlinear systems, Math. Systems Theory, 10 (1976), pp. 263-284.

[36] P. Tabuada and G. J. Pappas, Abstractions of Hamiltonian control systems, Automatica, J. IFAC, 39 (2003), pp. 2025-2033.

[37] P. Tabuada and G. J. PapPas, Hierarchical trajectory generation for a class of nonlinear systems, in Proceedings of the 42nd IEEE Conference on Decision and Control, Hawaii, 2003.

[38] P. Tabuada, G. J. Pappas, and P. Lima, Compositional abstractions of hybrid control systems, J. Discrete Event Dyn. Syst., 14 (2004), pp. 203-238.

[39] A. VAN DER SChaft, Symmetries and conservation laws for Hamiltonian systems with inputs and outputs: A generalization of Noether's theorem, Systems Control Lett., 1 (1982), pp. $108-115$.

[40] A. J. VAn Der Schaft And B.MaschKe, Interconnected mechanical systems, part I: Geometry of interconnection and implicit Hamiltonian systems, in Modelling and Control of Mechanical Systems, A. Astolfi, D. Limebeer, C. Melchiorri, A. Tornambe, and R. Vinter, eds., Imperial College Press, London, 1997.

[41] J. Willems, System theoretic models for the analysis of physical systems, Ricerche Automat., 10 (1979), pp. 71-106.

[42] W. M. Wonham, Linear Multivariable Control: A Geometric Approach, 2nd ed., SpringerVerlag, New York, 1979. 